\title{
Stock options y desempeño financiero en empresas cotizadas Mexicanas del sector industrial
}

\section{Stock options and financial performance on Mexican listed companies from industrial sector}

INFORMACIÓN DEL ARTÍCULO

Fecha de recepción: 16 de Octubre de 2018.

Fecha de aceptación: 29 de Noviembre de 2018.
1 Estudiante de la Maestría en Administración con énfasis en Negocios de la Universidad Autónoma de San Luis Potosí-México.

E-mail: wlomeli91@alumnos.uaslp.edu.mx Código ORCID:

https://orcid.org/0000-0003-2460-2703

${ }^{2}$ Doctora en Metodologías y Líneas de Investigación en Contabilidad y Auditoría. Docente e Investigadora de la Universidad Autónoma de San Luis Potosí-México

E-mail: guadalupe.briano@uaslp.mx

Código ORCID:

https://orcid.org/0000-0001-8241-0385

CITACIÓN: Lomelí Gómez, L.F., \& Briano Turrent, G.C. (2018). Stock options y desempeño financiero en empresas cotizadas Mexicanas del sector industrial. Podium, 34, 1-20. doi:10.31095/podium.2018.34.1

ENLACE DOI:

http://dx.doi.org/10.31095/podium.201 8.34 .1

Luis Fernando Lomeli Gómez ${ }^{1}$, Guadalupe del Carmen Briano Turrent ${ }^{2}$

\section{Resumen}

El objetivo de este estudio es analizar la relación que existe entre la adopción de las stock options y el rendimiento financiero en empresas del sector industrial que cotizan en la Bolsa Mexicana de Valores. De acuerdo a la teoría de agencia un esquema de compensación variable favorece la alineación de intereses entre los accionistas y los directivos, lo que lleva a alcanzar un mejor desempeño financiero. La muestra final de estudio integra 135 observaciones durante el periodo 2015-2017. Utilizando un análisis de regresión múltiple, los resultados empíricos muestran que las stock options no tienen influencia significativa en los indicadores de rentabilidad (ROA y ROE), sin embargo, incrementan el nivel de endeudamiento, lo que sugiere que motivan al equipo directivo a incrementar las oportunidades de inversión. Este estudio tiene implicaciones importantes para el diseño de nuevos esquemas de compensación en las empresas cotizadas en México.

\section{Palabras Clave:}

Stock Options, Gobierno Corporativo, Desempeño Financiero, Empresas Cotizadas, Sector Industrial, México.

Clasificación JEL: M51, G34.

\begin{abstract}
This study aims to analyze the relationship between stock options and financial performance of listed firms that belong to the industrial sector in the Mexican Stock Exchange. According to the agency theory, a variable compensation scheme may favor the interest alignment between shareholders and managers, leading to a higher financial performance. Our final study sample is composed of 135 observations made during the 2015-2017 period. Using a multivariate regression analysis, empirical results show that stock options do not influence significantly profitability indicators (ROA and ROE). However, the leverage level increases, which may motivate the management team to increase investment opportunities. This study has important implications for designing new compensation schemes for listed companies in Mexico.
\end{abstract}

Keywords:

Stock Options, Corporate Governance, Financial Performance, Listed Companies, Industry Sector, Mexico.

JEL Classification: M51, G34. 


\section{Introducción}

La competitividad en el mercado global ha originado nuevas formas de compensación para motivar a los empleados. Diversas organizaciones en la búsqueda de encontrar una ventaja competitiva, están adoptando nuevos esquemas de compensación con el objetivo de incrementar la productividad (Chang y Chen, 2002). La gestión de recursos humanos incide en el desarrollo, crecimiento, expansión y consolidación de una empresa. En la actualidad no basta con tener un buen producto o servicio, precios competitivos o reconocimiento en el mercado, sino un equipo de trabajo capaz de hacer frente a nuevos desafíos. La globalización trae más retos a las empresas, pues éstas deben de adaptarse a condiciones económicas globales $\mathrm{y}$ complejas y posicionarse en mercados locales, nacionales e incluso internacionales.

Los responsables de dirigir las corporaciones han tomado gran relevancia en los mercados y son objeto de análisis y observación. Por ello, las organizaciones han adoptado planes de incentivos que no solo buscan incrementar su productividad financiera sino también el retener a ejecutivos capaces, que impacten favorablemente en el resultado empresarial y reputación de la empresa.

Por su parte, el gobierno corporativo hace referencia a la adopción de mejores prácticas corporativas que garanticen un control y transparencia en el manejo de la empresa, razón por la cual, las prácticas de compensación han sido incluidas como un mecanismo disciplinador en la gestión gerencial. Latinoamérica se caracteriza por un sistema legal débil, una alta concentración accionaria y una escasa protección de los accionistas minoritarios. Estas condiciones llevan a las empresas a tomar decisiones en sus políticas de buen gobierno y financieras, como son la estructura de capital, políticas de dividendos, financiamiento y mecanismos de compensación y remuneración. La remuneración a ejecutivos constituye una herramienta de incentivo, cuyo objetivo es alinear intereses tanto de ejecutivos como de accionistas, procurando el logro de las metas establecidas por la empresa.

La remuneración variable representa un instrumento de gestión importante para alinear los intereses de accionistas y ejecutivos de la empresa. Es la Asamblea General de Accionistas y el Consejo de Administración los responsables de determinar los sistemas de remuneración del ejecutivo (Hodgson, 2009). En agosto de 2002, el Gobierno de los Estados Unidos de América (EE.UU.) presentó un informe sobre las remuneraciones de los consejeros en donde las empresas estaban obligadas a transparentar sus compensaciones. Estas prácticas y regulaciones se han extendido a otros países que han adoptado prácticas de remuneración variable e incrementado la transparencia de las políticas de sueldos y salarios, tales como EE.UU., Australia, Reino Unido, Francia, Alemania, Italia, España y Canadá. En América Latina existen tendencias que apuntan a este tipo de prácticas y han comenzado a adoptarse 
dentro de las empresas cotizadas. Por ejemplo, Brasil es el país más agresivo en incentivos a corto plazo, particularmente para las posiciones del Director General o Chief Executive Officer (CEO), por sus siglas en inglés, presidentes, vicepresidentes y miembros del consejo de administración (Thornton, 2015).

De acuerdo con Morgenson (1988) en el año de 1977, las 200 empresas más grandes de EE.UU. reservaban más del $13 \%$ de sus acciones ordinarias para planes de compensación variables. Los informes corporativos de las empresas en EE.UU. muestran que las prácticas de compensación variables constituyen aproximadamente el 55\% del salario del CEO en el $98 \%$ de las grandes compañías (Torres, 2012).

Ya son más las organizaciones que integran en sus sistemas de pago un componente variable de la retribución del personal. Esto se refiere a que además de recibir su salario fijo, las empresas optan por incentivar a sus trabajadores en base a sus resultados $y / 0$ metas que la organización pretende alcanzar (Romo, 2011). Incluso en algunos países anglosajones es obligatorio divulgar los mecanismos y montos de compensación en los estados financieros de las empresas (Gispert y Ortín, 2002). Las stock options tienen como objetivo alinear los objetivos del personal que ocupa cargos estratégicos con los objetivos corporativos. Una organización que busca consolidarse en un mercado competitivo debe contar con capital humano capaz de tomar decisiones efectivas, particularmente en los niveles superiores de la organización como son la posición de CEO, vice-presidentes $\mathrm{y} / \mathrm{o}$ directores henerales quienes impactarán en el ámbito financiero y económico de la empresa.

La teoría de agencia sustenta el uso de la compensación variable para incrementar la riqueza de la empresa (Sánchez y Aragón, 2002). Además, el plan de remuneración de ejecutivos deberá estar alineado al rendimiento y los niveles de riesgos de la empresa (Alfaro y Tribó, 2002). Las stock options disminuyen el riesgo de incurrir en un conflicto de interés por parte de los empleados y evitan las actitudes anti-éticas, y por consecuencia, la ocurrencia de un posible fraude (Gispert y Ortín, 2002).

El objetivo de este trabajo es identificar la relación que existe entre las prácticas de compensación variable, particularmente las stock options y el rendimiento financiero en empresas del sector industrial que cotizan en la Bolsa Mexicana de Valores (BMV). Para ello, se pretende responder a las siguientes preguntas de investigación: 1) ¿En qué porcentaje las empresas que cotizan en la BMV del sector industrial adoptan planes de compensación variable?, 2) ¿Las empresas cotizadas del sector industrial que han adoptado el esquema de las stock options alcanzan un mejor desempeño financiero en los indicadores de rentabilidad de ROA y ROE?, y 3) ¿Las empresas cotizadas del sector industrial que han adoptado el sistema de stock options disminuyen el nivel de endeudamiento de la empresa? 
Este trabajo se centra en las empresas del sector industrial que cotizan en la BMV durante el periodo 2015 - 2017. La muestra final se integra de 35 empresas cotizadas que pertenecen al sector industrial, dando un total de 135 observaciones. Este estudio contribuye a la literatura internacional, particularmente para el contexto latinoamericano. El tema de stock options no se ha abordado en México, por lo que constituye un tema innovador desde el punto de vista de la gobernanza corporativa. El código de gobierno corporativo en México, recomienda que el Consejo de Administración y la Asamblea de Accionistas revelen las políticas utilizadas y componentes que integran los paquetes de remuneración del CEO y de los funcionarios de alto nivel de la sociedad, por lo que el tema de compensación es de gran relevancia actual para las empresas cotizadas en Bolsa. Este trabajo analiza si el uso de planes de compensación variable impacta en el desempeño financiero, por lo que los resultados serán un referente para los responsables de diseñar los planes de compensación y para los accionistas de las empresas. Los resultados muestran que las stock options no tienen influencia significativa en los indicadores de rentabilidad (ROA y ROE), sin embargo incrementan el nivel de endeudamiento, lo que nos sugiere que motivan al equipo directivo a incrementar la inversión y favorecer el rendimiento a largo plazo.

El trabajo se estructura de la siguiente forma. En la segunda sección se desarrolla el marco teórico del trabajo. La tercera sección describe la metodología de investigación. La cuarta se refiere a los resultados del trabajo y la última sección concluye.

\section{Revisión de literatura}

Huselid (1995) sostiene que una gestión adecuada de recursos humanos, a través de un proceso eficiente de reclutamiento y selección, evaluación, y compensación disminuye la rotación de personal, incrementan la productividad y disminuyen los costos de capacitación del nuevo personal. Se ha encontrado evidencia del rol estratégico que tiene la compensación variable en la alineación de los intereses entre directivos y accionistas (Santone, Sigler y Britt, 1993).

De acuerdo con Rimsky (2005), la compensación variable proporciona al empleado la oportunidad de incrementar sus ingresos, siempre y cuando se logren las metas y objetivos establecidos por el consejo de administración y los accionistas. Al existir una alineación entre los objetivos del ejecutivo y los resultados de la empresa, el ejecutivo buscará la maximización de la utilidad. Sin embargo, es necesario que este tipo de planes cuenten con un sistema y procedimientos de auditoría externa y seguimiento de los resultados y que estén basados en las políticas financieras de la empresa y los intereses por parte de los accionistas para evitar conflictos de interés entre los accionistas y los directivos. Los planes de remuneración a ejecutivos en México en el corto plazo, están relacionados principalmente a los resultados en los estados financieros, 
referidos a indicadores como el Earnings Before Interest, Taxes, Depreciation and Amortization (EBTIDA), a las ventas y/o a los ingresos netos.

Las opciones sobre acciones se negociaron por primera vez el 26 de abril de 1973, en el Chicago Board Options Exchange (Bolsa de opciones de Chicago) y su crecimiento fue exponencial. Estas opciones sobre acciones se descubrieron sorpresivamente, aunque se conoce que ya habían sido negociadas silenciosamente por más de 100 años en el mercado fuera de la Bolsa. En la actualidad son consideradas como un instrumento adicional para la inversión y un medio de gestionar el riesgo para los inversionistas $y$ especuladores con experiencia (Little, 1991). Los estudios previos referidos a las remuneraciones de directivos a través de stock options tienen su origen en los años 80's. En la década de 1990, el valor agregado de las stock options otorgadas por las empresas estadounidenses aumentó más de 10 veces, de 11,000 millones de dólares en 1992 a 119,000 millones de dólares en el año 2000 (Murphy, 2003). Un estudio que se realizó a nivel mundial por Gallup reveló que México, es el país con mayor insatisfacción laboral de Latinoamérica, con solamente $12 \%$ de sus trabajadores completamente satisfechos con su empleo, $60 \%$ desconectados y un $28 \%$ que repudia su trabajo. Con respecto al resto de la región, Brasil tiene a los trabajadores más felices, y este país registra un $27 \%$. Aun así, el $62 \%$ de los trabajadores se sienten desacoplados y al $12 \%$ realmente no les gusta su trabajo
(Adams, 2013). Las stock options buscan alinear los intereses de ejecutivos y accionistas en la toma de decisiones y la retención de ejecutivos clave. Algunas de las empresas que sufren de liquidez han adoptado paquetes de remuneración agresivos basados en stock options como una alternativa viable para la atracción y motivación del capital humano (Shapiro y Stiglitz, 1984).

\section{Mercadeo de Capitales en México}

El "mercado" es un lugar o un área, dentro de los cuales los vendedores y compradores de una mercancía o servicio, mantienen y llevan a cabo transacciones mercantiles a un precio determinado (Monroy, 2000). Por su parte, el "mercado de dinero" es aquél en el que se llevan a cabo operaciones o transacciones mercantiles con activos financieros emitidos a plazo menor de un año, mientras que en el "mercado de capitales" se realizan operaciones o transacciones mercantiles emitidas a un plazo mayor de un año.

El mercado de capitales en México se encuentra conformado por 138 empresas cotizadas y que están inscritas públicamente a BMV, que a su vez están reguladas por la Comisión Nacional de Valores (CNV) para que el público inversionista, en general, deposite su dinero en la suscripción de los valores como instrumento de inversión.

El origen de la BMV se remonta en 1894, cuando Manuel Algara, Camilo Arriaga y Manuel Nicolín promovieron entre los más distinguidos corredores de 
comercio de la época, la idea de que la negociación de valores debía tener un marco normativo e institucional. Fue así como el 31 de Octubre de 1894 se fundó la Bolsa Nacional. En la actualidad es una institución privada constituida como sociedad anónima bursátil de capital variable, que opera bajo la concesión de la Secretaría de Hacienda y Crédito Público (SHCP) con apego a la Ley del Mercado de Valores. La BMV es el foro en el que se llevan a cabo las operaciones del mercado de valores en México, teniendo como objetivo facilitar las transacciones con valores y procurar el desarrollo del mercado, fomentando su expansión y competitividad (Bolsa Mexicana de Valores, 2017). La estructura de la BMV se divide en cuatro niveles de clasificación, incorporando un total de 10 Sectores, 24 Sub-Sectores, 78 Ramos y 192 Sub-Ramos. Dentro de la estructura del primer nivel se encuentra el Sector III que pertenece al sector industrial. De las 138 empresas que están listadas en el mercado de capitales mexicano, 35 pertenecen al sector industrial.

\section{Gobierno Corporativo}

El gobierno corporativo es el sistema adoptado para dirigir y controlar una corporación. El gobierno corporativo define los derechos y responsabilidades de los participantes corporativos clave, tales como: accionistas, junta directiva, funcionarios y administradores, y otras partes interesadas. Además, propone la estructura a través de la cual la empresa determina sus objetivos, desarrolla planes para lograrlos, y establece procedimientos para supervisar el rendimiento e implementar planes de incentivos para sus colaboradores (Gitman, 2007). El gobierno corporativo se ha centrado fundamentalmente en dos aspectos: (1) La composición óptima del consejo de administración, y (2) la creación del comité de auditoría (Fama 1980).

En este orden de ideas, el consejo de administración constituye una dimensión de gobierno corporativo que desempeña un rol supervisor en la actuación gerencial. De acuerdo con Rodríguez (2011), el consejo puede integrarse por accionistas mayoritarios y sus familias o por representantes de los accionistas. Otro caso es el de los consejos formados por gerentes de la misma empresa o por individuos externos a la propiedad y gestión. En otros contextos los consejos se componen de dos niveles, en los que además de la participación accionaria se integran a los órganos de toma de decisiones.

El CEO es el funcionario corporativo responsable de dirigir las operaciones diarias de la empresa e implementar y asegurar el cumplimiento de las políticas y estrategias encomendadas por el consejo de administración. Por su parte, el consejo de administración puede también proponer la conformación de comités de apoyo para garantizar el cumplimiento de las funciones. Tal es el caso del comité de nominación y compensación, cuya función se centra en el diseño e implementación de los planes de incentivos y compensaciones a susejecutivos para motivar al logro de 
objetivos y metas establecidos en la planeación estratégica, siendo el principal objetivo, incrementar la riqueza de los accionistas, el cual se mide por el precio y rendimiento de la acción.

\section{Código de Mejores Prácticas en México}

Las empresas cotizadas en México desempeñan un rol estratégico en la promoción del desarrollo económico y el progreso de la sociedad en México. Las empresas constituyen el motor de crecimiento y tienen la responsabilidad de generar riqueza, empleo, bienestar a la sociedad, infraestructura y bienes $\mathrm{y}$ servicios. El desempeño, la eficiencia, y la responsabilidad social son del interés público y privado, por lo tanto, el gobierno corporativo es una prioridad en la agenda nacional $\mathrm{y}$ en el sector empresarial.

En México, la Organización para la Cooperación y el Desarrollo Económicos (OCDE) es un organismo que regula una serie de normas establecidas por los países que conforman los organismos mundiales, del cual México forma parte. En 1999, la OCDE emitió los "Principios de la OCDE para el gobierno de las sociedades" los cuales se revisaron nuevamente en el año 2004. Dichos principios constituyen una referencia a nivel mundial para que cada país emita los propios, adecuándolos a su regulación y cultura empresarial. En este mismo año por iniciativa del Consejo Coordinador Empresarial (CCE) en México, se constituyó el comité de mejores prácticas corporativas, y se emitió el código de mejores prácticas corporativas en donde se establecieron una serie de recomendaciones o mejores prácticas para un mejor gobierno corporativo de las sociedades mexicanas. Para su edición se tomaron en cuenta las necesidades y características de las empresas mexicanas, como origen, estructura accionaria, sector industrial, entre otros. Lo que se procuró fue incrementar el nivel de competitividad de las sociedades mexicanas y que éstas pudieran acceder a diversas fuentes de financiamiento $\mathrm{e}$ incrementar el nivel de confianzas de los inversionistas nacionales e internacionales. $\mathrm{Si}$ bien estas recomendaciones no son obligatorias sino de carácter voluntario, sí se exige a las empresas que cotizan en bolsa, divulgar el nivel de adherencia y cumplimiento a este código, por lo que las empresas cotizadas en México han incorporado estas prácticas a su gestión, y algunas de ellas han diseñado sus propios códigos de buen gobierno, tomando como referencia el código general. El código de mejores prácticas en México se integra de 51 prácticas de buen gobierno, las cuales se agrupan en las siguientes dimensiones: 1) asamblea de accionistas, 2) consejo de administración, 3) función de auditoría, 4) función de evaluación y compensación, y 5) función de finanzas y planeación (Código de Mejores prácticas en México, 2010).

\section{Función de Evaluación y Compensación}

Las empresas consideran la necesidad de incentivar a sus trabajadores a través de esquemas de remuneración fija o variable. Generalmente los empleados que ocupan puestos estratégicos dentro de 
una organización, acceden a esquemas de remuneración variable. Resulta fundamental para los ejecutivos encontrar un equilibrio entre la compensación financiera y la compensación que no es meramente financiera sino más enfocada al clima laboral y a su satisfacción laboral. De acuerdo con Hernández (2000), un paquete de compensación eficaz en el contexto mexicano, se pudiera integrar por el sueldo, los incentivos y las prestaciones. El sueldo se refiere a la remuneración mensual en efectivo, sobre la base de un mes de trabajo. El incentivo se deriva de la compensación que reciben los trabajadores cuando se cumplen ciertas condiciones en los resultados financieros de la empresa. Y por último el concepto de prestaciones se utiliza para referirse a los pagos en efectivo adicionales al sueldo como son el aguinaldo, la prima vacacional, seguros médicos o de vida, etc., entre otras prestaciones en especie. Este paquete total de remuneraciones busca maximizar el aspecto motivacional de los trabajadores procurando alinear los intereses de trabajadores y accionistas.

El código de mejores prácticas en México recomienda apoyar al consejo de administración en la creación del comité de evaluación y compensación, que a su vez deberá diseñar criterios para designar o remover al director general y a los funcionarios de alto nivel, proponer los criterios para la evaluación y compensación del director general y los funcionarios de alto nivel, así como establecer las políticas y criterios para determinar los pagos y compensación de los consejeros. Por su parte, también es función de este comité, desarrollar la propuesta de la estructura y criterios para la compensación del personal, así como proponer al consejo de administración la aprobación del sistema formal de sucesión del CEO y funcionarios clave. Además las prácticas 39, 40 y 41 del Código de Mejores Prácticas Corporativas en México, recomiendan divulgar las políticas utilizadas y los componentes que integran los paquetes de remuneración del director general y de los cargos directivos (Código de Mejores Prácticas Corporativas en México, 2010).

\section{La remuneración variable en empresas del sector industrial de la Bolsa Mexicana de Valores}

El tema de la compensación o remuneración han sido objeto de debate constante en el sector empresarial. La evaluación de puestos que hasta hace pocos años era el pilar para administrar el sistema de compensaciones, pasó a ser parte de un sistema integral que incluye además de la evaluación del desempeño basado en objetivos, un sistema de compensaciones que determina estándares de medición y control para evaluar el desempeño (Segovia et al., 2011). De acuerdo con Kuhn (2009), los sistemas de compensación le dan forma a la cultura organizacional, inciden en la motivación, satisfacción y comportamiento de los empleados e impactan en el desempeño corporativo.

La compensación variable, también conocida con el nombre "pago de riesgo", es la que cambia directamente con el nivel de desempeño o resultados 
alcanzados en la organización (Madhani, 2010). De acuerdo con Gross y Bacher (1993), la compensación variable se puede definir como un plan de remuneración enfocado en el éxito de la organización que amplía las oportunidades de recibir incentivos a personas clave de la empresa. Las tendencias actuales en materia de compensación muestran una preferencia hacia la utilización de la compensación variable (Yockey, 2006). Esta tendencia fue confirmada por la encuesta de salarios realizada por Hewitt Associates en 2004, la cual mostró que el número de empresas que utilizaban al menos un tipo de compensación variable para personal no ejecutivo aumentó de $59 \%$ en 1995 a $80 \%$ en 2002.

En el caso de México, la figura 1 muestra que los ingresos de los CEO's en
México están conformados por un promedio de $48 \%$ en su salario base, un $23 \%$ en incentivos de corto plazo, y un $30 \%$ en incentivos de largo plazo. En la misma figura se muestra un comparativo con otros países de América Latina. Los incentivos de largo plazo que se observan en la figura 1, se basan en los resultados y en las utilidades de la organización. En México la compensación basada en acciones es poco común y no es exigible su registro contable en los estados financieros de la empresa.

Se pueden identificar tres formas habituales de programas de remuneración basados en acciones, que son normalmente ofrecidos a los ejecutivos: concesión de acciones, concesión de acciones con restricciones, y concesión de opciones sobre acciones.

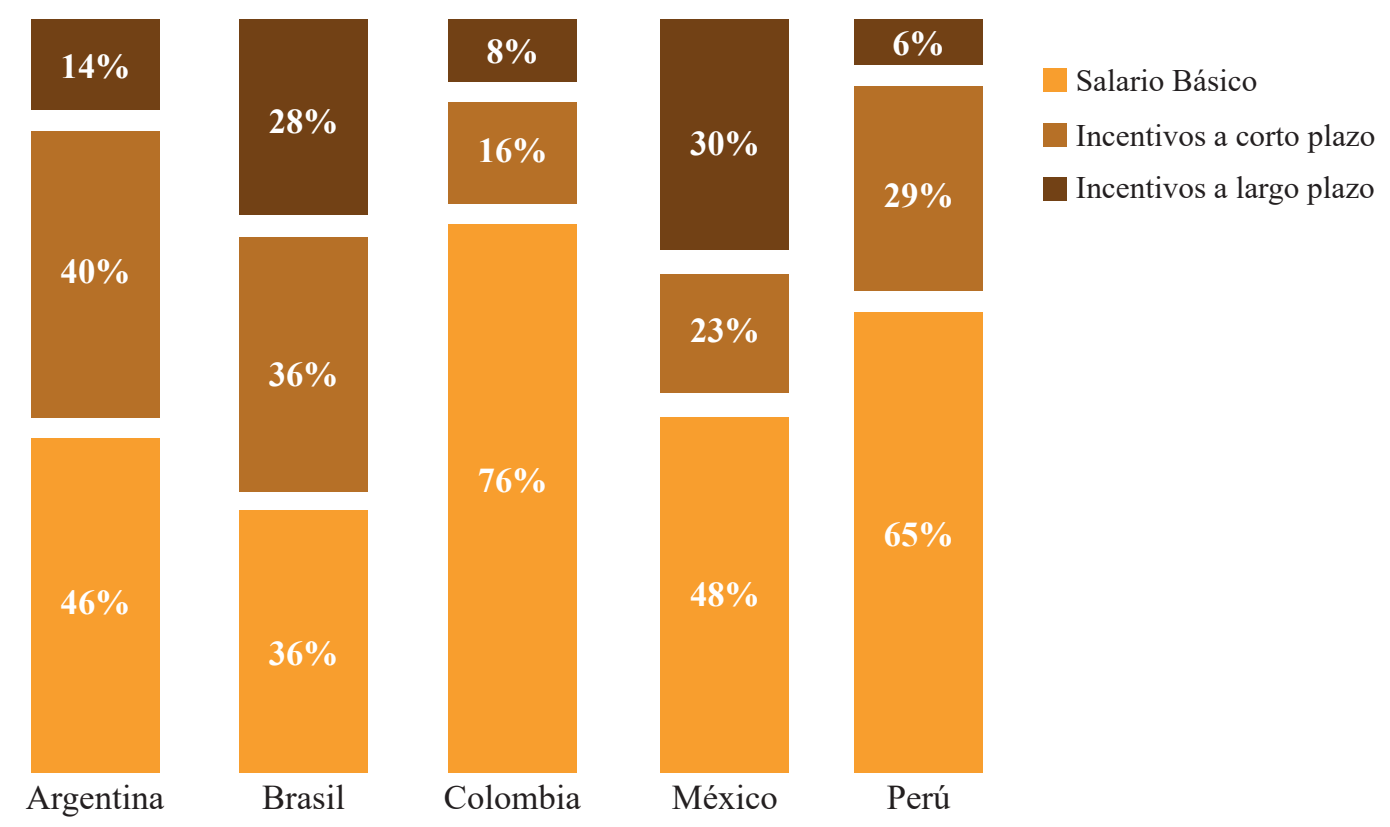

Figura 1. Mezcla de remuneración del CEO en Latinoamérica

Fuente: Encuestas Top Exec de Mercer (2014). 


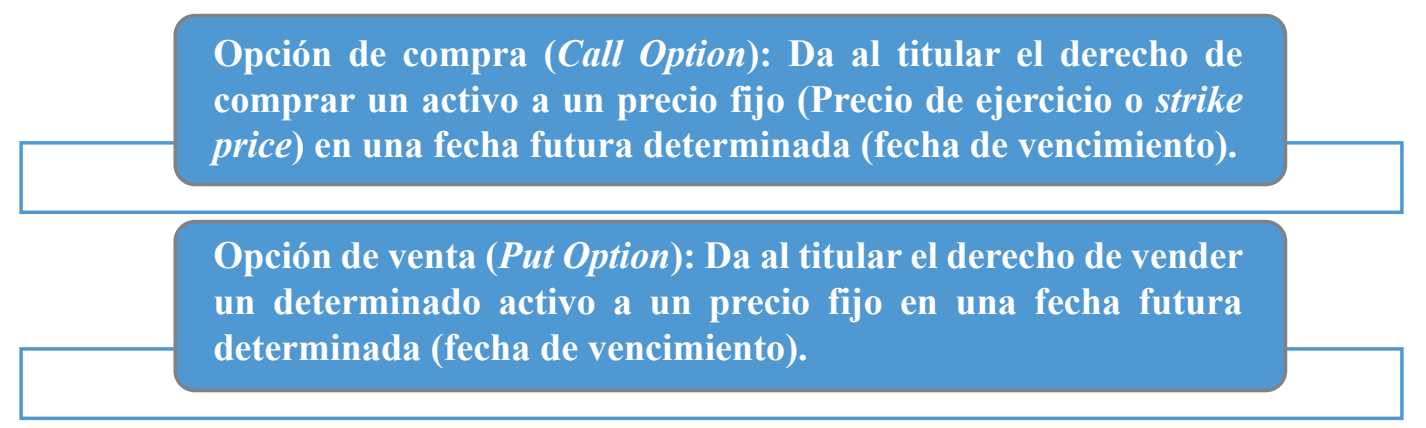

Figura 2. Tipos de opciones en el mercado financiero.

Fuente: Herbert (2008).

Para Herbert (2008), las opciones sobre acciones se definen como un contrato financiero que otorga al titular el derecho mas no la obligación, de ejecutar determinada operación en una fecha futura. O sea, el titular del derecho puede ejercer o no, la operación en la fecha establecida, conforme le parezca conveniente. Las características de este programa de remuneración variable, debe de derivar de los objetivos y de las estrategias trazadas por la organización. La mayor parte de los programas de remuneraciones por medio de las opciones sobre acciones, obliga al ejecutivo a continuar trabajando en la empresa durante un periodo para poder ejercer ese derecho en las utilidades. Los contratos de opciones sobre acciones son instrumentos de inversión considerados

Tabla 1.

Teorías sobre la Acción Colectiva

\begin{tabular}{|c|c|c|}
\hline Clasificación & Escenario & Descripción \\
\hline 1. In-the-money & $\mathrm{S}(\mathrm{t})>\mathrm{K}$ & $\begin{array}{l}\text { El precio de la acción }(\mathrm{S}) \text { en }(\mathrm{t}) \text { es mayor } \\
\text { al precio de ejercicio }(\mathrm{K}) \text {. }\end{array}$ \\
\hline 2. At-the-money & $S(t)=K$ & $\begin{array}{l}\text { El precio de la acción }(\mathrm{S}) \text { en }(\mathrm{t}) \text { es igual al } \\
\text { precio de ejercicio }(\mathrm{K})\end{array}$ \\
\hline 3. Out-of-the-money & $\mathrm{S}(\mathrm{t})<\mathrm{K}$ & $\begin{array}{l}\text { El precio de la acción }(\mathrm{S}) \text { en }(\mathrm{t}) \text { es menor } \\
\text { al precio de ejercicio }(\mathrm{K})\end{array}$ \\
\hline
\end{tabular}

Fuente: Kimura, Cruz-Basso y Perera (2009). como derivados, por depender o derivar de otros activos. En los mercados financieros existen dos tipos básicos de opciones: compra y venta. La figura 2 muestra las características de estos dos tipos de opciones.

Los contratos de opciones sobre acciones contienen los siguientes elementos: fecha de vencimiento $(t)$, precio de ejercicio $(\mathrm{k})$ y precio de la acción (S). Existen tres escenarios al momento de que el ejecutivo tiene el derecho de ejercer la compra o llegar a la fecha de vencimiento (t) del contrato, los cuales se muestran en la tabla 1. En el escenario 1 de la tabla 1, el ejecutivo ejerce la opción, paga el precio de ejercicio $(\mathrm{K})$ y recibe una acción que vale más $\mathrm{S}(\mathrm{t})$, en la cual el ejecutivo se vería beneficiado. En el 
escenario 2 y 3 de la misma tabla no valdría la pena ejercer la opción de compra ya que monetariamente no se vería beneficiado al comprar una acción que vale lo mismo en el escenario 2 o que vale menos en el escenario 3 , sin embargo, cabe mencionar que el funcionario tampoco se vería afectado ya que el ejercer la opción es un derecho y no una obligación (Kimura, Cruz-Basso y Perera, 2009).

\section{Ventajas y desventajas de las opciones sobre acciones}

La ventaja principal de las stock options es la alineación de intereses entre ejecutivos y accionistas, ya que el beneficio que tendría el ejecutivo va a derivar de los buenos resultados financieros de la empresa, y entre mayor sea el precio de las acciones, mayor serían las utilidades también del ejecutivo al depender del valor de éstas. Sin embargo, algunos analistas han señalado que las opciones sobre acciones pueden generar conflictos de interés entre directivos y accionistas (Jensen, 2004). Algunas empresas que han adoptado este esquema de remuneración a sus ejecutivos han llegado a sufrir fraudes por parte de sus ejecutivos al manipular los resultados financieros. Por lo anterior, es necesario que este tipo de esquemas de compensación estén auditados y que el comité de compensaciones asegure un manejo eficiente de los recursos financieros de la empresa.

\section{Hipótesis de estudio}

De acuerdo a la teoría de agencia, los administradores son considerados los representantes de los accionistas, quienes les delegan la autoridad para tomar decisiones dentro de la empresa. Al implementar los planes remuneración variable pueden existir problemas de agencia o conflictos de interés, siendo una posibilidad que los administradores antepongan sus metas personales, tales como riqueza personal, seguridad laboral, prestaciones adicionales, premios, entre otros. La relación entre principal-agente se basa en que el principal emplea al agente para que, mediante sus habilidades personales, materialice los objetivos en un contexto de incertidumbre y buen juicio (Knight, 1964). El conflicto de interés se manifiesta en decisiones de inversión, financiamiento, $y$ en las políticas de dividendos. Sin embargo, un mecanismo que pudiera minimizar los problemas de agencia consiste en integrar incentivos en los paquetes de remuneración de los directivos; siempre y cuando existan los mecanismos que inhiban posibles fraudes o manipulación contable, ya que como se ha demostrado en estudios previos, la compensación de capital gerencial está positivamente relacionada con el alcance de la manipulación de ingresos (Cheng y Warfield, 2005).

De acuerdo con Kohn (1998), las recompensas que pretenden incrementar el desempeño empresarial a largo plazo, tienen que ser continuas a través del tiempo. Las stock options se han convertido en un componente popular de la gestión de paquetes de compensación con el fin de alinear intereses y a su vez para que los directivos tomen las medidas 
necesarias para aumentar el precio de las acciones, y consecuentemente la riqueza de los accionistas. A medida que aumenta el precio de la acción, las stock options se vuelven más valiosas para los directivos, por lo tanto dan un incentivo para administrar e incrementar las ganancias.

Son diversos los estudios que muestran que las stock options mejoran la productividad y rentabilidad empresarial. Estos planes de incentivos promueven un monitoreo más eficiente (Hochberg y Lindsey, 2010; Kim y Ouimet, 2014), atraen a empleados más talentosos (Lazear, 1986), y retienen a los mejores empleados (Ittner, Lambert, y Larcker, 2003; Oyer, 2004). De acuerdo a una investigación realizada en empresas cotizadas en EE.UU, en donde se analizó la relación entre las stock options otorgadas a los cinco principales ejecutivos y las ganancias futuras; se concluyó que 1.00 usd del valor de una concesión de Stock Options, está asociado con el ingreso operativo futuro durante los próximos cinco años, de aproximadamente 3.71 usd (Hanlon, Rajgopal, y Shevlin, 2003). En la misma línea, otra investigación realizada en el Reino Unido, encontró una relación positiva y significativa entre el uso de las Stock Options y la productividad a nivel de empresa (Conyon y Freeman, 2004). En una muestra de empresas japonesas se estudió el impacto de las stock options sobre el rendimiento operativo de las empresa, encontrando que el rendimiento operativo posterior a la concesión de las acciones, es significativamente mayor en comparación con las empresas que no otorgan este tipo de incentivos (Kato et al., 2005). En el contexto de China, Lian, Su y Gu (2011), encontraron que el ROE mejoró en compañías que contaban con planes de incentivos, mientras que Fang, Nofsinger y Quan (2015) sustentan que las stock options incrementan el ROE en empresas cotizadas chinas.

En contraste Kanagaretnam, Mathieu y Ramanan (2009) utilizando datos de empresas de EE.UU, documentan una relación negativa entre las stock options y el rendimiento operativo. Del mismo modo, Melle (2005) evidencia una relación negativa entre las opciones sobre acciones y el rendimiento de la empresa para una muestra de empresas españolas y una muestra de empresas portuguesas, respectivamente. Dado lo anterior se establece la siguiente hipótesis.

H1. Las empresas cotizadas mexicanas del sector industrial que han adoptado planes de stock options para sus ejecutivos alcanzan una mayor rentabilidad.

La presencia de las opciones sobre acciones puede exacerbar o mitigar el papel disciplinario de la deuda. Jensen y Meckling (1976) afirmaron que la separación entre la propiedad y el control es una fuente esencial de los problemas de agencia. Ello llevó a que numerosos estudios como el de Mehran (1995), quien concluye que la propiedad en manos de los CEOs está positivamente asociada con el nivel de endeudamiento. Conforme incrementa el nivel de endeudamiento de una empresa, los directivos se sienten motivados para maximizar el valor del capital y seleccionar proyectos más rentables y por 
tanto tomar proyectos más riesgosos que podrían incrementar el nivel de endeudamiento (John y John, 1993). Por otro lado, la premisa de que el nivel de endeudamiento disminuye al implementar incentivos de tipo variable como las stock options, hace referencia a la fecha de ejercicio de las opciones sobre acciones, dado que el activo de la empresa podría incrementar su valor comparado con el nivel de endeudamiento, tomando así las acciones un valor positivo. Sin embargo, si ocurriese lo contrario, la empresa se encontraría con problemas en su situación financiera, ya que posiblemente el activo pasaría a manos de los acreedores y en ese caso los ejecutivos no ejercerían su opción de compra.

La estructura de capital debe estar bien definida ya que ahí es donde se reflejarán los costos de financiamiento y el control de propiedad de la empresa. Este costo es una de las variables más importantes al momento de tomar una decisión, procurando la maximización de las utilidades y obtener los recursos necesarios al menor costo. Dichos costos básicamente serían la tasa de interés o de descuento en el caso de la emisión de acciones, o en el caso de un crédito bancario este costo sería la tasa de interés del préstamo. De acuerdo a la discusión anterior se establece la siguiente hipótesis.

H2. Las empresas cotizadas mexicanas del sector industrial que han adoptado planes de stock options para sus ejecutivos incrementan el nivel de endeudamiento.

\section{Metodología de investigación}

\section{Descripción de la muestra de estudio}

El estudio es de carácter cuantitativo y se centra en las empresas del sector industrial que cotizan en la BMV. De las 146 empresas que cotizan en la BMV, únicamente se analizarán las empresas que pertenecen al sector industrial durante el periodo 2015-2017. El sector industrial es el que cuenta con un mayor número de empresas, las cuales representan el $24 \%$ del total de empresas cotizadas. La muestra final se integra de 35 empresas y 105 observaciones-año. La definición de la muestra, se consideró con respecto a las empresas mexicanas de capital abierto, específicamente del sector industrial, y se presenta en función de las variables necesarias para medir el impacto de la remuneración variable para ejecutivos sobre el desempeño financiero. Las variables de estudio han sido recolectadas de forma manual y se han obtenido de los informes anuales de cada una de las empresas analizadas y sus estados financieros. De las 105 observaciones obtenidas, únicamente el $34 \%$ de éstas otorgó un plan de remuneración basada en Stock Options, mientras que el $66 \%$ de la muestra optó por otro tipo de remuneraciones e incentivos a sus directivos.

\section{Variables de estudio}

Las variables de este estudio se han adoptado de la literatura previa que ha analizado el efecto de las stock options y el desempeño financiero en otros contextos. De acuerdo con Hanlon et al. (2003) y Bryan, Hwang, y Lilien (2000), las stock 
options mitigan los costos de agencia e incrementan el rendimiento financiero. Las variables dependientes se refieren al ROA, ROE y endeudamiento, mientras que las independientes se conforman por la decisión de adoptar las stock options como esquema de incentivos y las variables de control (tamaño de la empresa, edad de la empresa, sector industrial y año de estudio) (Hasewaga, Kim,y Yasuda, 2017; Yasuda, Kim, y Hasewaga, 2011; Smith y Watts, 1992).

\section{Modelo Estadístico}

Las ecuaciones 1 y 2 muestran los modelos de regresión a ser analizados para comprobar la relación entre el desempeño financiero (ROA, ROE y Endeudamiento) y el esquema de remuneración de stock options para los directivos (variable que toma el valor de 1, si la empresa adopta este esquema de remuneración y 0 en caso contrario). La tabla 2 presenta la definición de las variables de estudio.

$R O A / R O E=\alpha 0+\alpha 1$ StockOptions, $t+\alpha 2($ Control $)+\mu i .(1)$

Endeudamiento $=\alpha 0+\alpha 1$ StockOptions,$t+\alpha 2($ Control $)+\mu i$. (2)

En donde:

Control $=$ Variables de control como tamaño (log natural de los activos totales) y edad de la empresa (años desde su fundación).

$\mu_{i .}=$ Término de error.

Tabla 2.

Variables de estudio.

\begin{tabular}{ccc}
\hline Variable & Descripción & Medición \\
\hline $\begin{array}{c}\text { ROA (Return on Assets) } \\
\text { por sus siglas en Inglés } \\
\text { ó Retorno sobre los } \\
\text { Activos. }\end{array}$ & $\begin{array}{c}\text { Mide el rendimiento } \\
\text { obtenido con respecto al } \\
\text { activo total. }\end{array}$ & $\begin{array}{c}\text { ROA= Utilidad Neta / Activos } \\
\text { Totales }\end{array}$ \\
$\begin{array}{c}\text { ROE (Return on } \\
\text { en Inglés ó }\end{array}$ & $\begin{array}{c}\text { Muestra la rentabilidad que } \\
\text { reciben los accionistas } \\
\text { pentabilidad sobre el }\end{array}$ & ROE= Utilidad Neta / Total \\
Capital
\end{tabular}

Fuente: Hanlon et al. (2003); Bryan et al. (2000); Hasewaga et al. (2017); Yasuda et al. (2011); Smith y Watts (1992). 


\section{Resultados}

\section{Análisis Descriptivo}

La tabla 3 muestra las estadísticas descriptivas de las variables de estudio. Se observa que el ROA ha disminuido de un $10 \%$ en 2015 a un $5 \%$ en 2017 . El ROE por su parte ha pasado de un $18 \%$ en 2015 a un $7 \%$ en 2017. El nivel de endeudamiento también se ha reducido de un $76 \%$ a un $52 \%$. Con respecto a las opciones sobre acciones, se observa que las empresas han adoptado este esquema Tabla 3.

Estadísticos descriptivos de las variables de estudio. en el $34 \%$ de los casos y se ha mantenido durante el periodo de tiempo analizado.

\section{Análisis Correlacional}

La tabla 4 muestra las correlaciones entre las variables de estudio. Se observan relaciones significativas y positivas entre el ROE y ROA ( $\mathrm{p}=0.01)$, entre las opciones sobre acciones y el nivel de endeudamiento $(\mathrm{p}=0.05)$ y entre los años cotizados y la edad de la empresa $(\mathrm{p}=0.01)$.

\begin{tabular}{lccc}
\hline & $\begin{array}{c}\text { Muestra total } \\
(2015)\end{array}$ & $\begin{array}{c}\text { Muestra total } \\
(2016)\end{array}$ & $\begin{array}{c}\text { Muestra total } \\
(2017)\end{array}$ \\
\hline Variables & Media $\%$ & Media $\%$ & Media $\%$ \\
\hline ROA & 0.10 & 0.07 & 0.05 \\
ROE & 0.18 & 0.04 & 0.07 \\
Ende udamiento & 0.76 & 0.57 & 0.52 \\
Opciones sobre acciones & & & $34 \%$ \\
Sí & $34 \%$ & $34 \%$ & $66 \%$ \\
No & $66 \%$ & $66 \%$ & 9.95 \\
Tamaño de la empresa & 9.81 & 9.96 & 8.14 \\
Antigüedad & 8.35 & 8.58 & 35.56 \\
Años cotizando & 37.49 & 39.66 & \\
\hline
\end{tabular}

Fuente: Elaboración propia.

Tabla 4.

Matriz de correlación.

\begin{tabular}{|c|c|c|c|c|c|c|}
\hline Variable & $R O A$ & 1 & 2 & 3 & 4 & 5 \\
\hline 1. $R O E$ & $0.69^{* *}$ & & & & & \\
\hline 2. Endeudamiento & -0.12 & -0.01 & & & & \\
\hline 3. Opciones sobre acciones & -0.04 & -0.14 & $0.23^{*}$ & & & \\
\hline 4. Tamaño de la empresa & 0.01 & -0.04 & -0.09 & 0.05 & & \\
\hline 5. Antigüedad & -0.01 & 0.01 & -0.03 & -0.16 & 0.09 & \\
\hline 6. Años cotizados & -0.01 & 0.01 & -0.05 & $-0.30^{* *}$ & 0.05 & $0.75^{* *}$ \\
\hline
\end{tabular}

Fuente: Elaboración propia. 


\section{Análisis de Regresión}

La tabla 5 muestra el análisis de regresión entre las variables de estudio. La columna 1 muestra que no existe una incidencia significativa del esquema de las stock options sobre el indicador de rentabilidad ROA. Si bien en algunos estudios realizados para economías desarrolladas los resultados evidencian un incremento en la rentabilidad, pero el contexto mexicano es diferente y las stock options empiezan a cobrar relevancia en los mercados de capital. Lo mismo se observa para el ROE, en donde las stock options no tienen alguna influencia significativa. Dados los resultados anteriores la $\mathrm{H} 1$ se rechaza.

Tabla 5 .

Análisis de regresión múltiple.

\begin{tabular}{lccc}
\hline \multicolumn{1}{c}{ Variables } & $(1)$ & $(2)$ & $(3)$ \\
\hline Opciones sobre acciones & -0.01 & ROE & Endeudamiento \\
\hline Edad de la empresa & $(-0.15)$ & -0.34 & $0.38 * *$ \\
Tamaño de la empresa & 0.00 & $(-1.38)$ & $(2.28)$ \\
ROA & $(-0.13)$ & -0.01 & $(0.41)$ \\
Endeudamiento & 0.01 & $(-0.25)$ & -0.06 \\
Ronstante & $(0.06)$ & -0.02 & $(-1.07)$ \\
Año de estudio & & $(-0.29)$ & -0.13 \\
Cuadrado ajustado & -0.03 & 0.02 & $-1.35)$ \\
\hline
\end{tabular}

Fuente: Elaboración propia.
Por su parte, en el nivel de endeudamiento se observa que el esquema de stock options incrementa los niveles de endeudamiento $(\mathrm{p}=0.05)$, lo que va en línea con los resultados obtenidos por Mehran (1995), quien concluye que la propiedad en manos de los CEOs está positivamente asociada con el nivel de endeudamiento, dado que conforme incrementa el nivel de endeudamiento de una empresa, los directivos se sienten motivados para maximizar el valor del capital y seleccionar proyectos más rentables y por su aversión al riesgo disminuye lo que podría incrementar a su vez el nivel de endeudamiento. Por lo antes expuesto la H2 no se rechaza. 


\section{Conclusiones}

El presente estudio tuvo como objetivo analizar la relación entre el uso del esquema de las stock options a niveles directivos y el desempeño financiero (rentabilidad y endeudamiento) de empresas cotizadas mexicanas del sector industrial. Utilizando la teoría de agencia como fundamento teórico, la premisa de estudio se centró en afirmar que el esquema de stock options es efectivo en la función supervisora de la actuación gerencial, dado que motiva al agente (directivos) a alinearse a los objetivos de la organización, y por lo tanto, a mitigar los conflictos de agencia e incremento del desempeño financiero. Después de realizar los análisis estadísticos, se concluye que en el contexto de México, las stock options han sido adoptadas por el $34 \%$ de las empresas del sector industrial, lo que sugiere que aún falta camino por recorrer en este esquema de compensación, y por tanto, su incidencia en la rentabilidad no es significativa. Sin embargo, en el caso del nivel de endeudamiento, se observa un efecto favorable, lo que propone que las stock options como mecanismo de gobierno corporativo motivan a los directivos a incrementar el nivel de deuda, posiblemente para financiar proyectos de inversión más ambiciosos que beneficien a la empresa en sus resultados.

Esta investigación se limita a las empresas que cotizan en la BMV y que pertenecen al sector industrial, por lo que sería interesante para futuras investigaciones extender el estudio a todos los sectores de la BMV o incluir otros países. Además, sería relevante incluir otras variables financieras como es la toma de riesgos, pago de dividendos, gastos de inversión en innovación y desarrollo, así como las dimensiones del consejo de administración.

\section{Referencias}

Adams, S. (13 de Octubre de 2013). México, el país con mayor insatisfacción laboral de Latam. Obtenido de Forbes: https://www.forbes.com.mx/mexico-el-p ais-con-mayor-insatisfaccion-laboral-de-1 atam/\#gs.XTuqaS8

Alfaro, J.A. y Tribó, J.A. (2002). Rotación de la figura del director general $y$ consecuencias en la política laboral de la empresa. Cuadernos de Economía y Dirección de Empresa, 33, 373-386.

Bolsa Mexicana de Valores. (2017). Grupo BMV. Obtenido de :

http://www.bmv.com.mx/es/grupo-bmv/a cerca-de

Bryan, S., Hwang, L., y Lilien, S. (2000). CEO stock-based compensation: an empirical analysis of incentive-intensity, relative mix, and economic determinants. Journal of Finance, 73, 661-693.

Chang, P.L. y Chen, W.L. (2002). The effect of human resource management practices on firm performance: Empirical evidence from Taiwan's High-Tech firms. International Journal of Management, 19(4), 622-631.

Cheng, Q. y Warfield, T.D. (2005) Equity incentives and earnings management. The Accounting Review, 80(2), 441-476.

Código de Mejores Prácticas en México. Consejo Coordinador Empresarial. (2010). Consejo Coordinador Empresarial. Obtenido de: http://www.cce.org.mx/ 
Conyon, M. y Freeman, R. (2004). Shared modes of compensation and firm performance: UK Evidence. EconPapers. No. 8448. National Bureau of Economic Research, Inc.

Encuestas Top Exec de Mercer (2014). Obtenido de:file:///Users/gbriano/Downloads/Final _Webinar_Mercer_Tendencias_TopExec _LA_3006̄15.pdf.

Fama, E.F. (1980). Agency problems and the Theory of the Firm. Journal of Political Economy, 88(2), págs. 288-307.

Gispert, C. y Ortín, P. (2002). Mecanismos de disciplina de los directivos en España: el consejo de administración y los sistemas de retribución. Ekonomiaz, 50(2), 138-157.

Gitman, L.J. (2007). Principios de administración financiera. En L. J. Gitman, Principios de administración financiera (pág. 14). Ciudad de México: Pearson Educación de México, S.A de C.V.

Gross, S.E. y Bacher, J.P. (1993). The new variable pay programs: how some succeed, why some don't? Compensation and Benefits Review, 25(1), 51.

Hasewaga, N., Kim, H. y Yasuda, Y. (2017). Are executive stock options associated with future earnings? Journal of Accounting and Economics, 36 (1-3), 3-43.

Hanlon, M., Rajgopal, S. y Shevlin, T. (2003). The adoption of stock option plans and their effects on firm performance during Japan's period of corporate governance reform. Journal of The Japanese and International Economies, 44, 13-25.

Herbert, L.F. (2008). Stock Options e Criacao de Valor para o Acionista. Sao Paulo, Brasil: Inside Books.

Hernández, O.J. (2000). Guía para establecer un sistema de administración de la compensación en la empresa. Primera edición., México. Oxford University Press.

Hodgson, P. (October de 2009). A brief story of say on pay. Ivey Business Journal. available at: www.iveybusinessjournal.com/

Hochberg, Y.V. y Lindsey, L., 2010. Incentives, targeting, and firm performance. An analysis of non-executive stock options. Review of Financial Studies, 23, 41484186.

Huselid, M.A. (1995). The Impact of Human Resource Management Practices on Turnover, Productivity, and corporate financial performance. Academy of Managment, 38, 635-672.

Ittner, C.D., Lambert, R.A. y Larcker, D.F. (2003). The structure and performance consequences of equity grants to employees of new economy firms. Journal of Accounting and Economics, 34, 89-127.

Jensen, K. J. (2004). Remuneration: where we have been, how we got to here, what are the problems, and how to fix them. ECGI working paper series in finance, 44, 04-28.

Jensen, M.C. y Meckling, W.H. (1976).Theory of the firm: Managerial behavior, agency costs and ownership structure. Journal of Financial Economics, 3, 305-360.

Jensen, M.C. (2004). Agency costs of overvalued equity. Financial Managment. ECGI Finance working.

John, T.A. y John, K. (1993). Top-management compensation and capital structure. Journal of Finance, 48, 949-974.

Kanagaretnam, K., Mathieu, R. y Ramanan, R. (2009). Stock option grants, current operating performance and deferral of earnings. International Journal of Management, 26(1), 26-32. 
Kato, H., Lemmon, M., Luo, M. y Schallheim, J. (2005). An empirical examination of the costs and benefits of executive stock options: Evidence from Japan. Journal of Financial Economics, 78(2), 435-461.

Kim, E.H. y Ouimet, P. (2014). Broad-based employee stock ownership: motives and outcomes. Journal of Finance, 69, 1273-1319.

Kimura, H., Cruz-Basso, L.F., y Perera, L.C.J. (2009). Stock options e criação de valor para o acionista. Editota Atlas.

Knight, F.H. (1964). Risk, Uncertainty, and profit. En F. H. Knight, Risk, Uncertainty, and profit. New York: Reprints of Economic Classics.

Kohn, A. (1998). Punidos pelas recompensas. Sao Paulo: Atlas.

Kuhn, K.M. (2009). Compensation as signal of organizational culture: the effects of advertising individual or collective incentives. The International Journal of Human Resource Management, 20(7), 1634-1648.

Lazear, E.P. (1986). Salaries and piece rates. Journal of Business, 59, 405-431.

Lian, Y.J., Su, Z. y Gu, Y.D. (2011). Evaluating the effects of equity incentives using PSM: evidence from China. Frontiers of Business Research in China, 5, 266-290.

Little, J.B. (1991). Understanding Wall Street. Tab Books. Mc. Graw-Hill Inc.

Madhani, P.M. (2010). Rebalancing fixed and variable pay in sales organization: a business cycle perspective. Compensation y Benefits Review, 42(3), 179-189.

Melle, M. (2005). ¿Cómo valora el mercado de valores español la adopción de planes de opciones sobre acciones para directivos y consejeros? Investigaciones Económicas, XXIX (1), 73-115.

Mehran, H. (1995). Executive compensation structure, ownership, and firm performance. Journal of Financial Economics, 38, 163-184.

Monroy, J.D. (2000). El mercado de dinero y capitales y el sistema financiero mexicano. Ciudad de México: Editorial PAC, S.A de C.V.

Morgenson, G. (1988). Stock options are not a free lunch. Forbes. Obtenido de https://www.forbes.com/forbes/1998/051 8/6110212a.html

Murphy, B.J. (2003). The Trouble with Stock Options. The Journal of Economic Perspectives, págs. 49-70.

Oyer, P. (2004). Why do firms use incentives that have no incentive effects? Journal of Finance, 59, 1619-1650.

Rimsky, T.M. (2005). Administración de la Remuneración Total: Nuevos sistemas de pago al personal. Mexico, D.F. McGraw Hill Interamericana Editores.

Rodríguez, R.C. (2011). Los grupos económicos en México a partir de una tipología de arquitectura y gobierno corporativos: Una revisión de sus explicaciones teóricas. Fondo de Cultura Económica, LXXVIII(309), 193-234.

Romo, A.S. (2011). El rol de la remuneración variable en la efectividad organizacional de las empresas. International Journal of Good Conscience, 6(2), 17-33.

Sánchez, G. y Aragón, A. (2002). Retribución del directivo, factores contextuales y resultado de la empresa: Evidencia empírica en el sector químico-farmacéutico. Cuadernos de Economía y Dirección de Empresa, 12, 315-336. 
Santone, T.J., Sigler, K. J. y Britt, R. (1993). The strategic compensation planning process. Benefits Quarterly, 9, págs. 85-96.

Segovia, A., Mendoza, J., Rositas, J. y Abreu, J. (2011). El rol de la remuneración variable en la efectividad organizacional de las empresas. Daena: International Journal of Good Conscience, 6(2), 17-33.

Shapiro, C. y Stiglitz, J. (1984). Equilibrium employment as a worker discipline device. American Economic Review, 74, 433-444.

Smith, C.W. y Watts, R.L. (1992). The investment opportunity set and corporate financing, dividend, and compensation. Journal of Financial Economics, 32(3), 263-292.

Thornton, M.I.F. (2015). Remuneración de ejecutivos: Tendencias en América Latina. Remuneración de Ejecutivos: Tendencias en América Latina 2015, (pág. 30). Cd. de México.

Torres (2012). Planes de remuneraciones a través de acciones en su contexto contable, fiscal y laboral. Colegio de Contadores Públicos, A.C. Obtenido de Dinero e Imagen

http://www.dineroenimagen.com/2012-0 $8-06 / 4261$.

Yasuda, Y., Kim, H., y Hasewaga, N. (2011). Determinants of stock option adoptions in Japanese firms. Gendai Finance, 30, 27-59.

Yockey, M.D. (2006). Compensation policy as a signal of organization culture and of personal outcomes: Differential effects of individual incentive pay statements in recruitment advertising on attraction of experienced and inexperienced workers. (Tesis Doctoral). Recuperada de Proquest Dissertations y Theses. 